\title{
Física da microgravidade na sala de aula utilizando um Clinostato: uma revisão bibliográfica sistemática
}

\author{
Leonardo Amadeu de Sales, Rayssa Souza Lima Rodrigues, Victor José \\ Vasquez Otoya \& Diana Esther Tuyarot (i)
}

Instituto Federal do Sudeste de Minas Gerais - Campus Juiz de Fora, Rua Bernardo Mascarenhas 1283, Bairro Fábrica 36080-001, Juiz de Fora, Minas Gerais, Brasil. E-mail: diana.tuyarot@ifsudestemg.edu.br

Sales L.A., Rodrigues R.S.L., Otoya V.J.V. \& Tuyarot D.E. (2021) Física da microgravidade na sala de aula utilizando um Clinostato: uma revisão bibliográfica sistemática. Pesquisa e Ensino em Ciências Exatas e da Natureza, 5: e1784. http://dx.doi.org/10.29215/pecen.v5i0.1784

Editor acadêmico: Heydson Henrique Brito da Silva. Recebido: 21 junho 2021. Aceito: 01 setembro 2021. Publicado: 16 setembro 2021.

Resumo: Este trabalho é motivado pelo estudo dos conceitos de microgravidade e sua ocorrência no espaço, bem como poderia ser experimentada na superfície terrestre. O objetivo principal é explicar a física envolvida no fenômeno, em sala de aula, utilizando um Clinostato. Para sustentar o estudo foi realizada uma análise sistemática da literatura para elencar os artigos que tratem do tema e assim ter um panorama que facilite a compreensão do mesmo, e como poderia ser inserido em sala de aula. Para a análise foi utilizada a metodologia baseada em etapas, e as buscas foram realizadas em quatro plataformas: Periódicos CAPES; Google Acadêmico; Scielo e ERIC (Education Resources Information Center). Os descritores utilizados foram Microgravidade, Física, Clinostato e Ensino, em português e em inglês com abrangência de publicação entre 1995 e 2020. Os artigos encontrados foram analisados quanto ao tema de interesse no título e no abstract e foi realizada a leitura completa dos artigos selecionados. Poucos artigos foram encontrados voltados para o tema, isso chama a atenção em relação à atualidade do mesmo e à potencialidade para tratá-lo em caráter interdisciplinar.

Palavras chave: Formação de professores, Clinostato, novas metodologias, microgravidade simulada, tecnologia espacial.

\section{Physics of microgravity in the classroom: a systematic literature review}

Abstract: This work is motivated by the study of the concepts of microgravity and its occurrence in space as well as how it could be experienced on the Earth's surface. The main objective is to explain the physics involved in the phenomenon, in the classroom, using a clinostat. To support the study, a systematic analysis of the literature was carried out to select the articles that deal with the subject and therefore have a panorama that would help to comprehend the topic, as well as comprehend how it could be inserted in the classroom. For the analysis, the methodology based on stages was used, and the searches were carried out on four platforms: CAPES journals; Academic Google; Scielo and ERIC (Education Resources Information Center). The descriptors used were: Microgravity, Physics, Clinostat and Teaching, in Portuguese and in English, with publication coverage between 1995 and 2020. The articles found were analyzed regarding the topic of interest in the title and in the abstract, and a complete reading of the selected articles was carried out. Few articles were found on the topic, which drew attention in relation to its current nature and the potential to treat it in an interdisciplinary manner.

Key words: Teacher training, clinostat, new methodologies, simulated microgravity, space technology. 


\section{Introdução}

Há 50 anos da chegada do homem à Lua, e após a incursão no espaço do primeiro astronauta brasileiro, Marcos Pontes, que visitou a Estação Espacial Internacional (ISS, em inglês), em 2006, é importante que o conhecimento necessário para a conquista do espaço seja inserido na sala de aula.

Os conceitos físicos envolvidos na descrição da microgravidade têm sido estudados em várias situações e propostas. Por exemplo, no Guia de Atividades para Professores da National Aeronautics and Space Administration - NASA (Rogers et al. 1997) encontramos uma proposta que sugere introduzir o conceito na sala de aula mediante várias atividades de Ciência, Matemática e Tecnologia. Os autores do Guia explicam que as oportunidades de pesquisa em Física e Ciências Biológicas têm crescido bastante e observam que "poucas pessoas compreendem o conceito de microgravidade e como isto poderia ser utilizado para a pesquisa".

O Guia foi apresentado em 1997, no entanto, em 2017, Baliscei \& Rodrigues Jr (2017), 20 anos depois, afirmam que "o tema não faz parte do programa de Física para o Ensino Médio no Brasil, embora possa ser estudado como um caso especial da Força de Gravidade”. No trabalho de Baliscei \& Rodrigues Jr (2017), microgravidade em recintos fechados, três experiências são apresentadas utilizando a torre de queda livre, que é uma das propostas de situação de microgravidade na superfície terrestre (Baliscei \& Rodrigues Jr 2017). Anteriormente, Marcelo Saba do Instituto Nacional de Pesquisas Espaciais - INPE e colaboradores fizeram sugestões de introduzir o conhecimento em sala de aula (Saba et al. 2000). Para isso, ele e os colegas do Clube de Ciência Quark fizeram a proposta de várias experiências que poderiam ser realizadas para obter o ambiente de microgravidade em sala de aula. Atividades de baixo custo são escolhidas e realizadas com alunos do Ensino Médio. Nessas atividades são abordados temas de hidrostática, hidrodinâmica, magnetismo e oscilações, dentre outros (Saba et al. 2000).

A proposta do grupo de trabalho é estudar conceitos de microgravidade evidenciando a Física e mostrando, em sala de aula, como isso pode ser experimentado na Terra mediante a utilização de um Clinostato. Para isso está sendo construído um Clinostato 1D utilizando material de baixo custo. Resultados do projeto serão divulgados oportunamente. No que segue são apresentados a microgravidade e os tipos de Clinostato e seu funcionamento para embasar a motivação do trabalho.

\section{Microgravidade}

O termo "microgravidade" é utilizado para descrever a situação experimentada pelos astronautas na estação espacial. Também é utilizado para nomear a aceleração ( $g$ ') envolvida no ambiente de microgravidade, cuja magnitude é da ordem do milionésimo do valor da aceleração da gravidade da Terra.

No primeiro caso, os astronautas e os objetos na estação espacial experimentam uma ausência de peso devido ao fato de que se encontram em situação de queda livre (Rogers et al. 1997). Isto conduz, erroneamente, a pensar que a gravidade no espaço é zero. Tal afirmativa não é correta, pois o valor da aceleração da gravidade, g, vai diminuindo de forma quadrática com a distância. Quanto mais longe do planeta Terra, menor é a atração gravitacional, mas ela não chega a ser nula. Na altura da estação espacial, aproximadamente $400 \mathrm{~km}$, o valor de "g" é de 8.7 $\mathrm{m} / \mathrm{s}^{2}$.

A microgravidade corresponde a uma situação em que o peso aparente do sistema é pequeno se comparado ao peso real. Isso ocorre pelo fato de que a microgravidade, diferentemente da força gravitacional, produz uma ausência aparente de peso que permite que objetos e pessoas fiquem flutuando quando submetidos à ela, “... na ISS não se consegue a gravidade zero, restando fracos efeitos de gravidade, apropriadamente denominados de microgravidade." (da Silveira 2017). No trabalho de Saba et al. (2000), "A microgravidade na sala de aula", visa-se procedimentos de baixo custo e fácil reprodução, por exemplo, utilizando uma microcâmera e uma montagem simples (Figura 1). Estas experiências podem ser realizadas 
“pelos alunos e público em geral" (Saba et al. 2000). Os experimentos são submetidos a gravidade e a microgravidade a fim de observar como ambas influenciam, e assim poder entender melhor como a microgravidade funciona e quais são seus efeitos nos materiais e elementos.

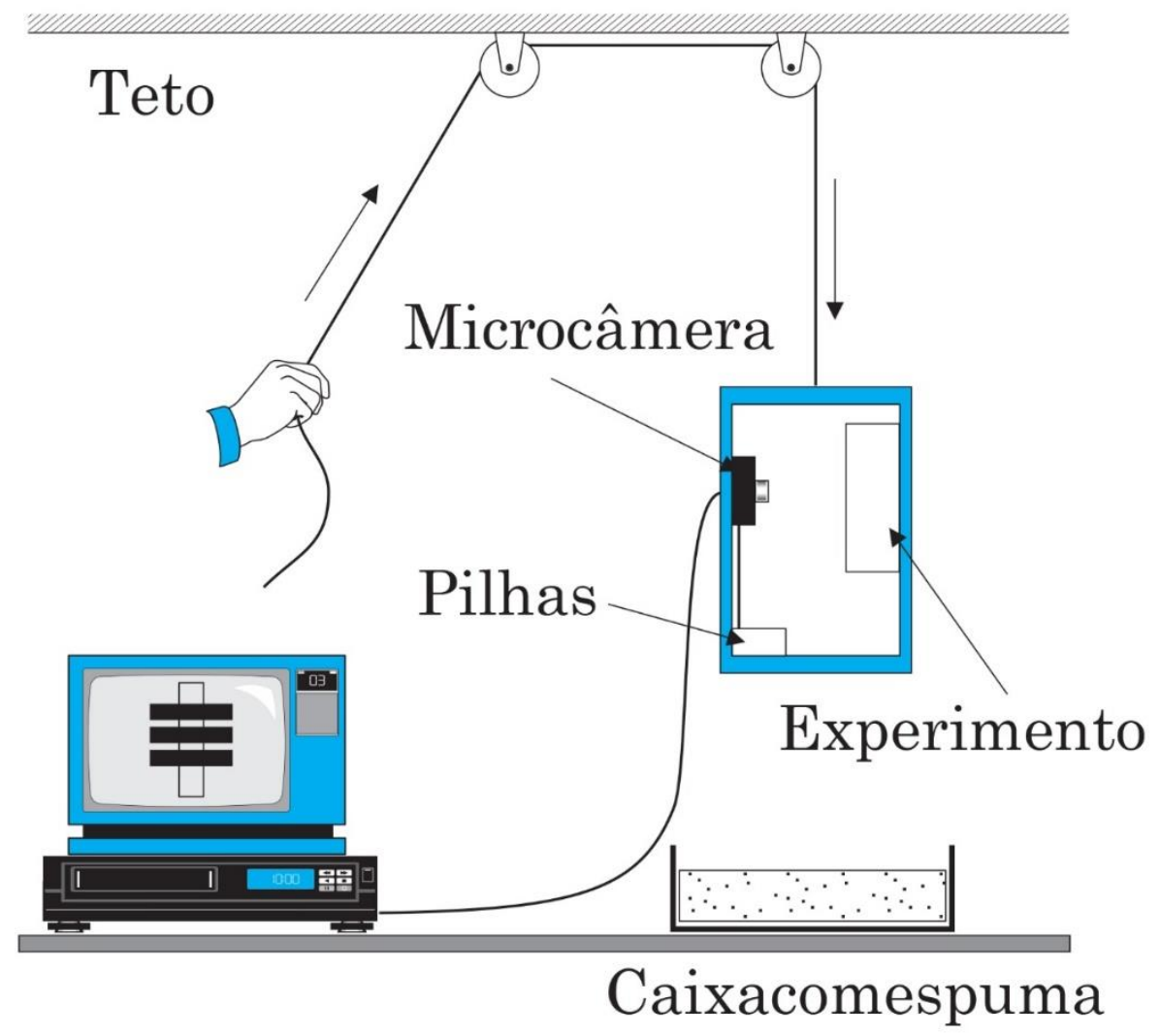

Figura 1. Equipamentos e montagem para experiências de microgravidade. Fonte: Saba et al. (2000).

Em relação à aceleração ( $\mathrm{g}$ ') envolvida, a microgravidade é, como foi dito, relativa ao valor da gravidade na superfície da Terra. No caso das experiências propostas, o ambiente de microgravidade é simulado com a queda livre. Outras experiências de baixo custo podem ser utilizadas em sala de aula, especificamente o Clinostato e outras máquinas baseadas no movimento de rotação.

Em geral, nas simulações de microgravidade utilizando máquinas de posicionamento aleatório (RPM, do inglês Random Position Machine), é levado em conta o valor da velocidade angular e do raio. Segundo Huijser (2000) isto leva à criação de um ambiente de microgravidade onde organismos vivos, tais como plantas, são bons candidatos para serem estudados devido à sua resposta.

Quando um corpo está submetido ao movimento de rotação constante, a aceleração assume, em $\left[\mathrm{m} / \mathrm{s}^{2}\right]$, um valor em torno de $10^{-4} \mathrm{~g}$ ou $10^{-5} \mathrm{~g}$, dependendo do raio do movimento e da velocidade angular (Figura 2). Nesta situação, o valor da aceleração centrípeta $a_{c p}=\omega^{2} r$ é relacionado com o valor da gravidade na superfície terrestre, $g=9.81 \mathrm{~m} / \mathrm{s}^{2}$. O fator de microgravidade é calculado conforme a equação (1) (Huijser 2000):

$$
g^{\prime}=\left(\omega^{2} r\right) / g
$$

onde, $\omega$ é a velocidade angular e $r$ o raio onde seria posicionado o corpo de teste para ser submetido a um determinado valor de microgravidade. 


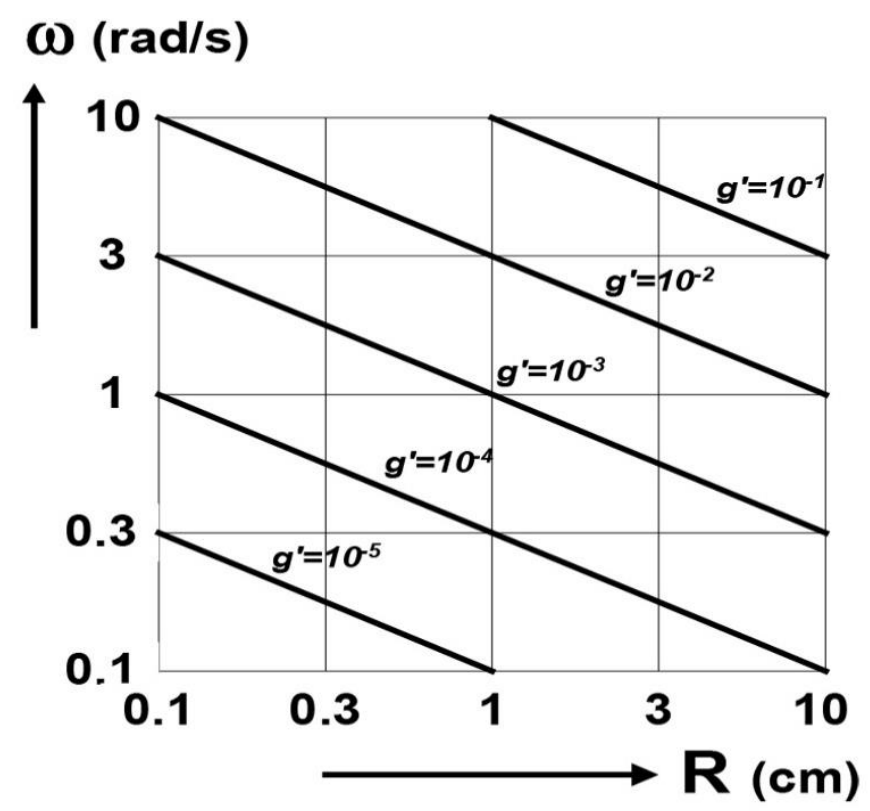

Figura 2. Gráfico Log-Log do raio e a velocidade angular. Fonte: Huijser (2000).

\section{O Clinostato}

Além das experiências evidenciadas pelos autores (Saba et al. 2000; Baliscei \& Rodrigues Jr 2017), também pode ser proposto para o estudo da física da microgravidade em sala de aula, o uso de um Clinostato. A invenção do Clinostato é atribuída a Julius von Sachs, que realizou estudos de tropismos em plantas (Rafferty 2007). O estudo de fenômenos como, por exemplo, o gravitropismo (resposta à gravidade) e seus efeitos em organismos vivos tais como plantas, resulta do interesse no avanço da ciência espacial. Esta experiência proporciona uma oportunidade de trabalho interdisciplinar ao aluno, evidenciando sua importância.

O Clinostato trata-se de um aparelho criado para estudo do comportamento de plantas. Utilizado na botânica, o Clinostato teve posteriormente seu uso estendido à Biologia em geral. Hoje é utilizado para o estudo de células cancerosas e outros fenômenos. Os primeiros Clinostatos tiveram uma versão unidimensional (1D), e com as novas tecnologias estes foram evoluindo até chegar à uma versão 3D, na qual o aparelho tem três eixos de rotação e giro (Laván \& Valdivia-Silva 2015).

O Clinostato funciona rotando a uma velocidade angular uniforme, cujos valores estão entre 2 e $4 \mathrm{rpm}$ para evitar efeitos de centrifugação na amostra. Além da rotação, este possui uma força de atrito entre a amostra e o meio dentro da placa. Este movimento, de rotação, cria um ambiente onde a amostra está submetida a uma frequência. Com isto, o Clinostato poderá simular o ambiente de microgravidade quando a frequência é escolhida adequadamente (Albrecht-Buehler 1992). O Clinostato 1D possui somente um eixo de revolução (Figura 3A) e os chamados 3D possuem três eixos, um chamado eixo de rotação, outro eixo de revolução e, por fim, o eixo desorientador (Figura 3B) (de Oliveira Alves 2013). Ainda podem ser encontradas as "Random Position Machine - RPM" que tem movimento randômico utilizado como base o design do Clinostato como mostrado na Figura 3C.

$\mathrm{Na}$ literatura são encontradas várias propostas de uso do Clinostato voltadas para atividades de Biologia em sala de aula (Dietlein et al. 2013; Programa Profundiza Andaluzia 2016) ou pesquisas específicas voltadas para Biologia ou ciência espacial (Zhang et al. 2016; Lionheart et al. 2018; Chantseva et al. 2019). No entanto, a proposta aqui é ensinar os conceitos de Física envolvidos na experiência.

Após esta breve apresentação, que motivou o trabalho, será apresentada a metodologia para uma revisão da literatura focalizando "o ensino de microgravidade na sala de aula de Física utilizando um Clinostato". 
Física da microgravidade em sala de aula

As perguntas que incentivam a pesquisa são:

- Quais autores estudam microgravidade usando um Clinostato?

- Quais autores evidenciam os conceitos físicos?

- Quais autores têm o propósito de inserir os mesmos em sala de aula?
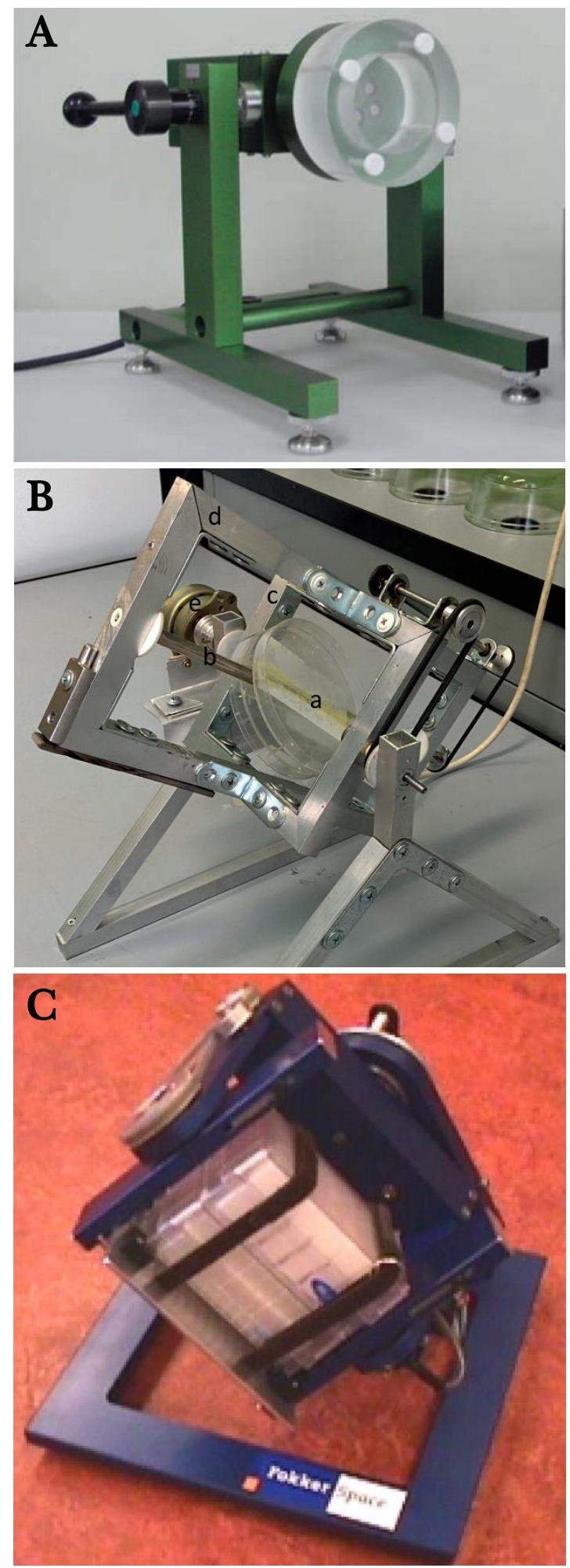

Figura 3. Modelos de Clinostatos com eixo de revolução, rotação, desorientador e design com movimento randômico: A. Clinostato 1D (Dietlein et al. 2013); B. Clinostato 3D (Chantseva et al. 2019); C. RPM (Huijser 2000). 


\section{Metodologia}

O roteiro para a revisão sistemática foi composto a partir dos trabalhos de Kitchenham (2004) e Conforto et al. (2011) que apresentam as razões pelas quais realizar uma busca sistemática. Para uma busca mais aprimorada, Conforto et al. (2011) apresentam um método de Revisão Bibliográfica Sistemática - RBS Roadmap, dividido em fases e etapas.

Fase 1 - Após a formulação do problema e objetivos, foi realizada a pesquisa nas fontes. Inicialmente, como se pretende estudar a microgravidade e seus efeitos, a pesquisa incluiu vários artigos relacionados com o tema (Saba et al. 2000; Dietlein et al. 2013; Programa Profundiza Andaluzia 2016; Zhang et al. 2016; Baliscei \& Rodrigues Jr 2017; Lionheart et al. 2018) o que levou a um critério de busca de material baseado nos descritores, relacionados mais adiante, e uma lista relevante de periódicos que poderiam melhor balizar o trabalho (Tabela 1). Isto determinou a fase 1 do trabalho.

Uma busca sistemática na literatura foi realizada no portal de periódicos CAPES, na base Scielo, no Google Acadêmico e na plataforma ERIC (Education Resources Information Center) dos últimos 25 anos, com os descritores "microgravity" e "clinostat" e "physics" e "teaching" para artigos em inglês ou outros idiomas; e "microgravidade” e "Clinostato" e "Física” e “ensino” para revistas em português.

Tabela 1. Lista de periódicos e banco de dados consultados.

\begin{tabular}{|c|c|c|}
\hline $\begin{array}{c}\text { Classificação CAPES } \\
\text { Quadriênio 2013-2016 }\end{array}$ & Título & ISSN \\
\hline $\begin{array}{l}\text { A1: Ensino } \\
\text { B1: Educação } \\
\text { B1:Interdisciplinar } \\
\text { B5: Astronomia / Física; }\end{array}$ & Revista Brasileira de Ensino de Física & Versão impressa: 1806-1117 \\
\hline $\begin{array}{l}\text { B2: Ensino } \\
\text { B3: Interdisciplinar }\end{array}$ & Física na escola & Versão impressa: 1983-6422 \\
\hline $\begin{array}{l}\text { B2: Ensino } \\
\text { C: Astronomia/Física }\end{array}$ & Física na escola & Versão eletrônica: $1983-6430$ \\
\hline $\begin{array}{l}\text { B1: Ensino } \\
\text { B4: Interdisciplinar }\end{array}$ & Revista de Enseñanza de la Física & Versão impressa: 0326-7091 \\
\hline $\begin{array}{l}\text { A2: Ensino } \\
\text { B2: Educação } \\
\text { C: Astronomia / Física; } \\
\text { Ciências Biológicas II }\end{array}$ & Caderno Brasileiro de Ensino de Física & Versão impressa: 1677-2334 \\
\hline $\begin{array}{l}\text { A1: Ensino } \\
\text { B1: Interdisciplinar }\end{array}$ & Enseñanza de las ciencias & Versão impressa: 0212-4521 \\
\hline A1: Ensino & Teaching and Teacher Education & Versão impressa: 0742-051X \\
\hline Banco de dados & Periódicos CAPES & \\
\hline Banco de dados & Google Acadêmico & \\
\hline Banco de dados & Scielo & \\
\hline Banco de dados & ERIC & \\
\hline
\end{tabular}

Na busca no Portal de Periódicos CAPES com a entrada "microgravidade" foram encontradas 43 publicações das quais somente 01 tinha o tópico relacionado ao ensino de Física, os autores tratam microgravidade em recintos fechados (Baliscei \& Rodrigues Jr 2017). Com a entrada "microgravidade e física" foram encontradas 04 publicações, das quais somente 02 correspondem a artigos avaliados por pares. Um dos artigos é o anteriormente referenciado, na busca nas revistas, e o outro trata de Fisiologia, motivo pelo qual foi excluído da busca. Quando acrescentado o descritor "Clinostato" na combinação nenhuma publicação foi achada. O mesmo aconteceu quando foi acrescentado "ensino".

Continuando no portal CAPES, com os descritores em inglês, inseridos um a um, foram encontrados 23.146 artigos revisados por pares em todos os tópicos fornecidos pela base de 
dados. Um refinamento com o critério de inclusão pelo tópico "physics" apresenta 3.165 artigos. Já quando inseridos os descritores combinados "microgravity; physics; teaching" foram encontrados 63 artigos dos quais após separados pelos critérios ficaram 03 (Galili 1995; Hershey 2005; Temiz \& Yabuz 2014). Acrescentando "clinostat", e separados pelos critérios de ser artigo que trate o tema: microgravidade, Física e ensino de Física usando um Clinostato (microgravity; physics; clinostat; teaching), foram encontrados 2 itens, um artigo voltado para o ensino de tropismos (Hershey 2005) e arquivos de um symposium voltados para área de Fisiologia. Nenhum artigo foi encontrado na área de Física.

$\mathrm{Na}$ base Scielo, na busca geral por microgravidade apresenta 04 artigos, 02 voltados para corpo humano, 01 para Botânica e 01 para Física (Baliscei \& Rodrigues Jr 2017). Não foram encontrados artigos com a combinação dos descritores. Em inglês foram encontrados 20 artigos para "microgravity" sendo somente 03 voltados para a área de Física, 01 para simulação numérica (Mendes \& Cucchieri 2006), 01 aplicado a sistemas dinâmicos (Cappellari et al. 2005) e o artigo de Microgravity Indoor de Baliscei citado anteriormente (Baliscei \& Rodrigues Jr 2017). A combinação de descritores encontra novamente o artigo de simulação numérica e nenhum artigo com "clinostat" e "teaching".

No Google Acadêmico em português, para o descritor "microgravidade", foram encontrados um total de 906 trabalhos incluídas citações e 803 excluídas citações, no período 1995-2020. Dentre estes foram selecionados 30 trabalhos que tratam de experiências; ensino; histórico; clube de ciências; livro paradidático; jogos; filmes e um voltado para patente, todos relacionados na Tabela 2. Outras áreas e tópicos tratados tais como: Engenharia; Robótica; Medicina; Biologia; Fisiologia; Farmácia; Ligas; Arquitetura; Ambiente; Literatura e Poesia não foram analisadas por não abranger nosso critério de revisão.

Quando inseridos os descritores "microgravidade" e "Física" os resultados encontrados superpõem com os anteriores. Já com "microgravidade e Física e Clinostato" 06 artigos são encontrados todos voltados para área biológica, os quais foram excluídos. Nenhum artigo é encontrado com todos os descritores voltado para o objetivo do trabalho (microgravidade, Física e ensino de Física usando um Clinostato).

No caso do Google Acadêmico em inglês, a busca por "microgravity; physics; clinostat; teaching" não apresenta trabalho voltado para o objetivo. Os resultados tratam de Biologia Experimental, Fisiologia, ossos, Farmacologia, bactérias. Não foi encontrado trabalho voltado para o ensino de Física em outra língua diferente do português nesta plataforma.

Na base ERIC com o descritor em inglês "microgravity", há 33 trabalhos dos quais foram separados 25 voltados para o ensino do tópico em vários níveis escolares todos relacionados na Tabela 3. Os trabalhos foram organizados por tópicos similares aos dos encontrados no Google Acadêmico com a intenção de comparar os tópicos da Tabela 2 com os tópicos da Tabela 3. Analisando a Tabela 3 foi evidenciado que, de 24 trabalhos feitos, apenas 01 utiliza o Clinostato. Para a combinação de descritores a base apresenta 05 trabalhos dos quais somente 01 usa $\mathrm{o}$ Clinostato que foi encontrado anteriormente e, em outra base, o trabalho é voltado para ensino de Biologia (Hershey 2005).

Fase 2 - Leitura dos resumos dos artigos selecionados com a finalidade de organizar os mesmos com os objetivos. Foram separados os artigos que, no título ou no resumo, tratam de ensino de Física ou experiência usando microgravidade, além de outros como: histórico; clube de ciências; livro paradidático; jogos; filmes e voltado para patente.

Foram encontrados 01 artigo do portal CAPES em português (Baliscei \& Rodrigues Jr 2017) e 03 artigos na busca em inglês (Galili 1995; Hershey 2005; Temiz \& Yabuz 2014). Na base Scielo, somente 01 para Física foi encontrado [novamente o artigo de Baliscei \& Rodrigues Jr (2017)]. Na plataforma Google Acadêmico foram separados 30 trabalhos (Tabela 2) encontrados na busca em português e nenhum resultado em inglês. Por último, na base ERIC, em inglês, 25 trabalhos foram encontrados (Tabela 3).

Os tópicos escolhidos para as tabelas são os mesmos para comparar a produção em português e inglês. Com isso podemos perceber que a grande maioria dos trabalhos é voltada 
para experiências/demonstrações ou ensino. Na produção em inglês, os trabalhos provêm principalmente da NASA.

Com isto, pode-se observar que, embora vários trabalhos apresentem o tópico "microgravidade", os autores focalizam o ensino ou aplicação em Ciências Biológicas, engenharias e Farmacologia, dentre outros, e não na Física. No caso dos que tratam de ensino ou aplicação na Física, os mesmos não têm como foco principal ensinar a Física envolvida e sim, na maioria deles, os resultados do fenômeno no arranjo. As experiências propostas são principalmente a torre de queda livre e a experiência do elevador. Além de trabalhos que apresentam estudo de foguetes e plataforma de lançamento. Outros trabalhos que tratam da Física, o fazem de forma histórica ou como análise de conteúdo de livro didático. Já os trabalhos que relacionam microgravidade, Física e ensino foram mantidos, assim como alguns dos que trazem o uso do Clinostato nesse contexto.

Tabela 2. Resultados da base Google Acadêmico separados por tópicos. Todas as produções (30 trabalhos) estão em português.

\begin{tabular}{lcl}
\hline \multicolumn{1}{c}{ Tópicos } & Quantidade & \multicolumn{1}{c}{ Artigos } \\
\hline Experiências/demonstrações & 7 & $\begin{array}{l}\text { Wasiliew (2002); Novelo (2016); Baliscei \& Rodrigues Jr } \\
\text { (2017); Colares (2018); Dantas et al. (2018); da Silva (2019); } \\
\text { Dutra \& Souza (2019); }\end{array}$ \\
\hline Ensino & 11 & $\begin{array}{l}\text { Saba } \text { et al. (2000); da Silva (2002); Figueiredo (2005); Reis \& } \\
\text { Garcia (2006); Lima (2008); Tenório et al. (2014); Sousa } \\
\text { (2015); Vasconcelos \& Marranghello (2015); Borragini } \\
\text { (2016); Silva (2018); Rodrigues (2019); }\end{array}$ \\
\hline Histórico & & $\begin{array}{l}\text { Sausen (1999); Macau (2007); Rodrigues (2012); da Silva } \\
\text { (2013); Pesquero (2015); }\end{array}$ \\
\hline Clube de ciências & 5 & Passoni (2011); \\
\hline Livro Paradidático & 1 & Moura (2017); \\
\hline Jogos & 1 & Knechtel (2008); Ribeiro \& Galera (2009); \\
\hline Filmes/ vídeos & 2 & Morais (2015); Borragini et al. (2017); \\
\hline Patente & 2 & Dos Santos et al. (2016). \\
\hline
\end{tabular}

Tabela 3. Resultados da base ERIC separados por tópicos. Todas as produções (25 trabalhos) estão em inglês.

\begin{tabular}{lcl}
\hline \multicolumn{1}{c}{ Tópicos } & Quantidade & \multicolumn{1}{c}{ Artigos } \\
\hline Experiências/ demonstrações & 20 & Vogt \& Wargo (1992, 1995); Mohler (1997); Williams (1997); \\
& & Rogers \& Wargo (1998); Casaburri \& Gardner (1999); LaCombe \& \\
& & Koss (2000); NASA3 (2000); Alshibli1 (2002); Alshibli2 (2002); \\
& & Vreeland (2002); NASA1 (2003); NASA2 (2003); Hershey (2005); \\
& & Banerjee et al. (2008); Matthews et al. (2012); Dou et al. (2013); \\
& & Dittrich (2014); Secco \& Sukara (2016); Schappe \& Barbosa \\
& & (2017); \\
\hline Ensino & 4 & Chandler (1991); Rogers et al. (1997); NASA4 (1999); An (2016); \\
\hline Histórico & 0 & - \\
\hline Clube de ciências & 0 & - \\
\hline Livro Paradidático & 0 & - \\
\hline Jogos & 0 & - \\
\hline Filmes/ vídeos & 1 & NASA5 (2002). \\
\hline Patente & 0 & - \\
\hline
\end{tabular}

Fase 3 - Leitura dos artigos selecionados

Na sequência, foi realizada a leitura iniciando pelos artigos encontrados no portal CAPES, Scielo e os relacionados nas Tabelas 2 e 3. O artigo de Galili (1995) trata de preconcepções acerca do conceito leveza, Hershey (2005) propõe uma experiência para o estudo de tropismos usando um Clinostato, e Temiz \& Yavuz (2014) trata acerca da Segunda Lei de Newton no espaço extraterrestre. Como já observado, Hershey (2005) usa o Clinostato, mas o objetivo é ensinar Biologia e não a Física envolvida. O restante dos artigos tem a "microgravidade" sendo utilizada 
como embasamento principal e, como já foi observado, a maioria estão voltados para experiência/demonstrações ou ensino.

Os trabalhos foram reorganizados de acordo com as perguntas, assim, a partir do conjunto encontrado em relação com os questionamentos resulta:

\section{(1) Que autores estudam microgravidade usando um Clinostato?}

Hershey (2005) estuda o tópico, microgravidade utilizando um Clinostato, mas o trabalho é voltado para ensino de Biologia. No vídeo apresentado na seção do Clinostato, Laván \& Valdivia-Silva (2015) estudam, desde o ponto de vista da Astrobiologia, os efeitos da gravidade nos sistemas biológicos. Nas referências (Vogt \& Wargo 1992; Rafferty 2007; Dietlein et al. 2013; Dos Santos et al. 2016; Novelo 2016; Programa Profundiza Andaluzia 2016; Zhang et al. 2016; Lionheart et al. 2018) o tópico também é desenvolvido, alguns buscando aperfeiçoar o aparelho e desenvolver patentes, sem, no entanto, evidenciar os conceitos físicos - um total de (10/69; $14 \%$ ) nos últimos 25 anos.

\section{(2) Quais autores evidenciam os conceitos físicos?}

Em relação com os conceitos físicos, os mesmos são evidenciados nos trabalhos empregados nesta pesquisa (Rogers et al. 1997; Saba et al. 2000; Baliscei 2016; da Silveira 2017), nos artigos (Cappellari 2005; Mendes \& Cucchieri 2006) de formas tais como simulação numérica e sistemas dinâmicos e outros que apresentam experiências, sequências didáticas, tratamentos matemáticos e físicos (Vogt \& Wargo 1992, 1995; Rogers \& Wargo 1998; NASA4 1999; NASA3 2000; Wasiliew et al. 2002; Figueiredo 2005; Macau 2007; da Silva 2013; Schappe \& Barbosa 2017; Colares 2018; da Silva 2019) - um total de (18/69; 26\%) dos trabalhos analisados.

\section{(3) Quais autores têm o propósito de inserir os mesmos em sala de aula?}

Inserção em sala de aula é tratado pelos autores (Galili 1995; Vogt \& Wargo 1995; Rogers et al. 1997; Williams 1997; Casaburri \& Gardner 1999; Sausen 1999; Saba et al. 2000; Alshibli2 2002; da Silva 2002; NASA5 2002; NASA2 2003; Cappellari 2005; Reis \& Garcia 2006; Knechtel 2008; Lima 2008; Ribeiro \& Galera 2009; Passoni et al. 2011; Matthews et al. 2012; Rodrigues et al. 2012; Dietlein et al. 2013; Dou et al. 2013; Dittrich 2014; Temiz \& Yavuz 2014; Tenório et al. 2014; Morais 2015; Pesquero 2015; Sousa 2015; Vasconcelos \& Marranghello (2015); An et al. 2016; Baliscei 2016; Borragini 2016; Secco \& Sukara 2016; Borragini 2017; Moura 2017; da Silva 2018; Dutra \& Souza 2019; Rodrigues 2019); isto é realizado em diversos níveis de ensino, desde Ensino Fundamental até formação continuada de professores. Nestes trabalhos tem experiências, sequências didáticas, problematização, interpretação de conceitos, dentre outros, focalizando a sala de aula. O universo de trabalhos tem um total de $(38 / 69 ; 55 \%)$ lembrando que parte é voltada para a sala de aula de Biologia.

No trabalho de da Silva (2018) é realizada uma análise da ocorrência do termo "microgravidade" nos livros didáticos de Física do $1^{\circ}$ ano do Ensino Médio em relação com concepções espontâneas do conceito segundo a autora: "é possível afirmar que o assunto aqui tratado carece de mais textos que tenham como objetivo esclarecer e desmistificar a ideia de gravidade zero muitas vezes utilizada de uma forma equivocada". Com isto, é possível voltar ao tema que motivou a pesquisa sistemática e é razoável embasar a mesma mostrando que existe uma carência na literatura acerca do tema proposto, focalizando o ensino de microgravidade na sala de aula de Física utilizando um Clinostato.

\section{Considerações finais}

Neste trabalho é apresentada uma revisão bibliográfica sistemática da literatura a partir da busca sistemática baseada no método RBS Roadmap, dividido em fases e etapas de Conforto et al. (2011). Esta pesquisa revisou vários artigos e trabalhos em periódicos nas bases Periódicos CAPES; Google Acadêmico; Scielo e ERIC. Com isto foi possível apresentar um panorama dos 
trabalhos voltados para a compreensão do conceito de microgravidade, sua ocorrência no espaço e quais são as experiências que são propostas na literatura para reproduzir o fenômeno na superfície terrestre e apresentar o conceito em sala de aula. $\mathrm{O}$ critério utilizado foi de ser artigo que trate o tema microgravidade, Física e ensino de Física usando um Clinostato e pretendeu-se responder a três questões norteadoras: 1) que autores estudam microgravidade usando um Clinostato? 2) quais autores evidenciam os conceitos físicos? e 3) quais autores têm o propósito de inserir os mesmos em sala de aula?

A partir da análise dos trabalhos foi constatado que existem poucos artigos voltados para o tema, o que chama a atenção em relação com sua atualidade e a potencialidade para tratá-lo em caráter interdisciplinar. A proposta de apresentar a Física envolvida no fenômeno da microgravidade, utilizando a experiência do Clinostato, em sala de aula está em andamento e resultados serão apresentados futuramente. $\mathrm{O}$ estudo de fenômenos como, por exemplo, o gravitropismo (resposta à gravidade) e seus efeitos em organismos vivos, tais como plantas, aponta o interesse para o avanço da ciência espacial. A experiência de uso do Clinostato proporciona oportunidade de trabalho interdisciplinar ao aluno evidenciando sua importância.

\section{Agradecimentos}

Os autores agradecem aos avaliadores pela contribuição, à CAPES e a instituição pelo financiamento.

\section{Referências}

Albrecht-Buehler G. (1992) The Simulation of Microgravity Conditions on the Ground. American Society for Gravitational and Space Biology Bulletin, 5(2): 3-10.

Alshibli1 K. (2002) Using Space for a Better Foundation on Earth: Mechanics of Granular Materials. Educational Brief. Grades 5-8. Disponível em: https://files.eric.ed.gov/fulltext/ED463 178.pdf (Acessado em: maio/2020).

Alshibli2 K. (2002) Using Space for a Better Foundation on Earth: Mechanics of Granular Materials. Educational Brief. Grades 9-12. Disponível em: https://files.eric.ed.gov/fulltext/ED46 3179.pdf (Acessado em: maio/2020).

An S.A., Zhang M., Tillman D.A., Robertson W., Siemssen A. \& Paez C.R. (2016) Astronauts in Outer Space Teaching Students Science: Comparing Chinese and American Implementations of Space-to-Earth Virtual Classrooms. European Journal of Science and Mathematics Education, 4(3): 397-412.

Baliscei M.P. \& Rodrigues Junior A.A. (2017) Microgravity indoor. Revista Brasileira de Ensino de Física, 39(2): e2502. https://doi.org/10.1590/1806-9126-rbef-2016-0230

Banerjee S., Andring K., Campbell D., Janeski J., Keedy D., Quinn S. \& Hoffmeister B. (2008) Orbital Motion of Electrically Charged Spheres in Microgravity. Physics Teacher, 46: 460464. https://doi.org/10.1119/1.2999060

Borragini E.F. (2016) O ensino de astronomia na formação continuada de professores com ênfase na gravitação universal. Dissertação (Programa de Pós-Graduação em Ensino de Física). Universidade Federal do Rio Grande do Sul. Instituto de Física, Porto Alegre.

Borragini E.F., Pavani D.B. \& Lima Jr. P. (2017) Gravitação Universal em atividades práticas: uma abordagem histórica e cultural, das órbitas dos planetas à ficção científica. Textos de apoio ao professor de Física. Volume 28, Número 1. Porto Alegre: UFRGS. 83 p.

Cappellari F.O., Lifschitz L.A., Brito H.H. \& Maglione L.S. (2005) ¿Qué se publica hoy en Iberoamérica? Información Tecnológica, 16(2): 53-60.

Casaburri A.A. \& Gardner C.A. (1999) Space Food and Nutrition: An Educator's Guide with Activities in Science and Mathematics. Washington, DC: National Aeronautics and Space Administration.

Chandler D. (1991) Weightlessness and Microgravity. Physics Teacher, 29(5): 312-313. 
Chantseva V., Bilova T., Smolikova G., Frolov A. \& Medvedev S. (2019) 3D-clinorotation induces specific alterations in metabolite profiles of germinating Brassica napus L. seeds. Communications Biology, 64(1): 55-74. https://doi.org/10.21638/spbu03.2019.107

Colares D.M. (2018) Criação de um minilevitador acústico ultrassônico de baixo custo como produto educacional no ensino de Física. Monografia (Licenciatura em Física). Universidade Federal do Ceará, Centro de Ciências, Fortaleza.

Conforto E.C., Amaral D.C. \& da Silva S.L. (2011) Roteiro para revisão bibliográfica sistemática: aplicação no desenvolvimento de produtos e gerenciamento de projetos. $8^{\circ}$ Congresso Brasileiro de Gestão de Desenvolvimento de Produto. Porto Alegre: UFRGS.

Dantas F.C., Taveiros F.E.V., Dantas J.B.D. \& De Carvalho Z.V. (2018) PD\&I Aeroespacial: análise da implementação de uma plataforma hipersônica de lançamento orbital no centro de lançamento da barreira do inferno. Proceeding of ISTI/SIMTEC, 9(1): 446-454. https://doi.org/10.7198/S2318-3403201800010051

da Silva H.C. (2002) Discursos escolares sobre gravitação newtoniana: textos e imagens na física do Ensino Médio. Tese (Programa de Pós-Graduação em Educação). Universidade Estadual de Campinas, Faculdade de Educação, Campinas.

da Silva M.F. (2013) O Programa Espacial Brasileiro em perspectiva histórica: do início a 2010. Parcerias Estratégicas, 18(37): 195-208.

da Silva G.B. (2018) Uma análise sobre a ocorrência do termo microgravidade em livros didáticos do $1^{\circ}$ ano do ensino médio. Monografia (Curso de Licenciatura em Física). Instituto Federal de Educação, Ciência e Tecnologia do Rio Grande do Norte, Santa Cruz, Rio Grande do Norte.

da Silva V.B. (2019) Sequência didática para o ensino de microgravidade com utilização de um aparato experimental. Dissertação (Mestrado Nacional Profissional em Ensino de Física). Universidade Federal Rural do Semiárido, Mossoró, Rio Grande do Norte.

da Silveira F.L. (2017) Microgravidade é o mesmo que gravidade nula? Centro de Referência para o Ensino de Física. Disponível em: https://www.if.ufrgs.br/novocref/?contactpergunta=microgravidade-e-o-mesmo-que-gravidade-nula (Acessado em julho/2020).

de Oliveira Alves K. (2013) Desenvolvimento de um clinostato tridimensional com três eixos de rotação que permite a troca de suportes de amostras. Dissertação (Programa de PósGraduação em Engenharia Elétrica). Pontifícia Universidade Católica do Rio Grande do Sul, Faculdade de Engenharia, Porto Alegre, Rio Grande do Sul.

Dietlein I., Doi T., Haubold H., Hauslage J., Hemmersbach R., Hoson T., Lammens S., Li Y., Long M., van Loon J.J.W.A., Niu A., Takahashi H., Ochiai M., Osman A. \& Steffens H. (2013) Programme on Space Applications: Teacher's Guide to Plant Experiments in Microgravity. New York: English, Publishing and Library Section, United Nations Office at Vienna. 56 p.

Dittrich W.A. (2014) Drop Tower Physics. Physics Teacher, 52(7): 415-417.

Dos Santos M.A., Russomano T., Forbes B. \& Bosquillon C. (2016) Câmara para a difusão de ingredientes ativos, e processo para o cultivo de células em microgravidade ou microgravidade simulada. Patente. Disponível em: https://www.researchgate.net/profile/Thais_ Russomano2/publication/303913309_Camara_para_a_difusao_de_ingredientes_ativos_e_process o_para_o_cultivo_de_celulas_em_microgravidade_ou_microgravidade_simulada/links/575d216 a08aec91374abccc6.pdf (Acesso em: maio/2020).

Dou R., Hogan D., Kossover M., Spuck T. \& Young S. (2013) Defusing Diffusion American. Biology Teacher, 75(6): 391-395.

Dutra C.M. \& Souza M. (2019) O uso da problematização e do pêndulo simples para o estudo da gravidade. Revista Thema, 16(1): 10-23.

Figueiredo J.M.L. (2005) É tempo de dizer eureka! no ensino de Física. Disponível em: https://arxiv.org/ftp/physics/papers/0503/0503002.pdf (Acessado em: maio/2020).

Galili I. (1995) Interpretation of students' understanding of the concept of weightlessness. Research in Science Education, 25: 51-74.

Hershey D.R. (2005) Time for a Plant Clinostat: effects of light and gravity on plants. Science Activities, 42(1): 30-35. https://doi.org/10.3200/SATS.42.1.30-35 
Huijser R.H. (2000) Desktop RPM: New Small Size Microgravity Simulator for the Bioscience Laboratory.FS-MG-R00-017 @ Fokker Space. Downloaded form the DESC web site: http://www. desc.med.vu.nl

Kitchenham B. (2004) Procedures for performing systematic reviews. Keele, UK: Keele University. $26 \mathrm{p}$.

Knechtel C.M. (2008) O lúdico no Ensino de Ciências. Monografia (Programa de Desenvolvimento de Formação Continuada dos Profissionais da Educação do Estado do Paraná). Universidade Estadual do Oeste do Paraná, Núcleo de Cascavel, Paraná.

LaCombe J.C. \& Koss M.B. (2000) The Make-It-Yourself Drop-Tower Microgravity Demonstrator. Physics Teacher, 38(3): 143-146.

Laván D. \& Valdivia-Silva J. (2015) Astrobiología: la máquina de microgravedad peruana, INICTEL - UNI. Disponível em: https://www.youtube.com/watch?v=bmxvMKoENi0 (Acesso em: maio/2020).

Lima M.S. (2008) As Ciências Espaciais, o saber e o fazer docente no ensino fundamental. Dissertação (Programa de Pós-Graduação em Ensino de Ciências Naturais e Matemática). Universidade Federal do Rio Grande do Norte, Natal, Rio Grande do Norte.

Lionheart G., Vandenbrink J.P., Hoeksema J.D. \& Kiss J.Z. (2018) Microgravity. Science and Technology, 30: 491-502.

Macau E.E.N. (2007) Chegamos à Lua (p. 75-122). In: Almeida P.A.F.B. \& Winter O.C. (Orgs). A conquista do Espaço: do Sputnik à Missão Centenário. São Paulo: Editora Livraria da Física. $221 \mathrm{p}$.

Matthews K.R., Motiwala S.A., Edberg D.L. \& García-Llama E. (2012) Flight Mechanics Experiment Onboard NASA's Zero Gravity Aircraft. Journal of Technology and Science Education, 2(1): 4-12.

Mendes T. \& Cucchieri A. (2006) Numerical Simulation of N-vector Spin Models in a Magnetic Field. Brazilian Journal of Physics, 36(3): 648-651.

Mohler R.R.J. (1997) Space Shuttle Experiments Take Flight. Science and Children, 35(3): 26-30.

Morais V.D. (2015) O uso filmes cinematográficos no Ensino de Física: uma proposta metodológica. Monografia (Graduação em Licenciatura em Física). Faculdade de Educação e Meio Ambiente, Ariquemes, Rondônia.

Moura F.P. (2017) Utilização de um livro paradidático no processo de ensino e aprendizagem de conceitos de física. Dissertação (Programa de Pós-Graduação em Ensino de Física). Universidade Federal Rural do Semi-Árido, Mossoró, Rio Grande do Norte.

NASA1 - National Aeronautics and Space Administration. (2003) Wireless Drop Tower for Microgravity Demonstrations. Educational Brief. Disponível em: https://files.eric.ed.gov/fullte xt/ED479516.pdf (Acessado em: maio/2020).

NASA2 - National Aeronautics and Space Administration. (2003) Science in a Box: An Educator Guide with NASA Glovebox Activities in Science, Math, and Technology. Disponível em: https://files.eric.ed.gov/fulltext/ED479519.pdf (Acessado em: maio/2020).

NASA3 - National Aeronautics and Space Administration. (2000) A NASA Recipe for Protein Crystallography. Educational Brief. Disponível em: https://files.eric.ed.gov/fulltext/ED481752. pdf (Acessado em: maio/2020).

NASA4 - National Aeronautics and Space Administration. (1999) Microgravity: Fall into Mathematics. Educational Brief. Disponível em: https://files.eric.ed.gov/fulltext/ED460852.pdf (Acessado em: maio/2020).

NASA5 - National Aeronautics and Space Administration. Langley Research Center. (2002) Measurement, Ratios, and Graphing: Who Added the "Micro" to Gravity? An Educator Guide with Activities in Mathematics, Science, and Technology. NASA CONNECT[TM]. EG-2002-1014-LaRC Disponível em: https://files.eric.ed.gov/fulltext/ED472181.pdf (Acessado em: maio/2020).

Novelo L. (2016) Aperfeiçoamento do Clinostato 3D e seu uso no estudo dos efeitos da microgravidade em sementes de milho. Dissertação (Programa de Pós-Graduação em 
Engenharia Elétrica). Pontifícia Universidade Católica do Rio Grande do Sul, Faculdade de Engenharia, Porto Alegre, Rio Grande do Sul.

Passoni S., Mayer N., Colman J., Brinatti A.M., Rutz da Silva S.L. \& Borges da Silva J. (2011) Vamos viajar ao espaço? Revista Conexão UEPG, 7(2); 162-167.

Pesquero L.C. (2015) Proposta de um minicurso de Astronomia para alunos do Ensino Fundamental II. Dissertação (Programa de Pós-Graduação em Ensino de Ciências Exatas). Universidade Federal de São Carlos, Centro de Ciências Exatas e de Tecnologia, São Carlos, São Paulo.

Programa Profundiza Andaluzia (2016) proyecto "Germinación y crecimiento de plantas en microgravedad simulada" realizado por alumnos y alumnas de $3^{\circ}$ y $4^{\circ}$ ESO del IES " $\mathrm{M}^{\mathrm{a}}$ Victoria Atencia” de Santa Rosalía - Maqueda (Málaga). Plantas en Gravedad Simulada. Disponível em https://plantasenmicrogravedad.wordpress.com/2015/05/23/nuevas-versionesde-clinostato/ (Acessado em: abril/2020).

Rafferty J.P. (2007) Julius von Sachs. German Botanist. Encyclopaedia Britannica. Disponível em https://www.britannica.com/biography/Julius-von-Sachs\#ref34694 (Acessado em: abril/2020).

Reis N.T.O. \& Garcia N.M.D. (2006) Educação espacial no Ensino Fundamental: uma proposta de trabalho com o princípio da ação e reação. Revista Brasileira de Ensino de Física, 28(3): 361371.

Ribeiro R.J. \& Galera J.M.B. (2009) Jogos epistêmicos para educação. In: I Simpósio Nacional de Ensino de Ciência e Tecnologia. 1356 p.

Rodrigues M.C.S. (2019) A ABP como estratégia didática e a astronomia como contexto no ensino da quantidade de movimento. Dissertação (Programa de Pós-Graduação em Ensino de Física). Universidade Federal de Sergipe, São Cristóvão, Sergipe.

Rodrigues E.V., Zimmermann E., \& Hartmann Â.M. (2012) Lei da gravitação universal e os satélites: uma abordagem histórico-temática usando multimídia. Ciência \& Educação, 18(3): 503-525. http://dx.doi.org/10.1590/S1516-73132012000300002

Rogers M.J.B., Vogt G.L. \& Wargo M.J. (1997) Microgravity: A Teacher's Guide with Activities in Science, Mathematics, and Technology. Washington, DC: National Aeronautics Space Administration. $168 \mathrm{p}$.

Rogers M.J.B. \& Wargo M.J. (1998) The Microgravity Demonstrator. NASA EG-1998-12-49-MSFC. Disponível em: https://files.eric.ed.gov/fulltext/ED448051.pdf (Acessado em: maio/2020).

Saba M., Silva B.B. \& de Paula P.R.J. (2000) Microgravidade em Sala de Aula. Física na Escola, 1(1): 15-17.

Sausen T.M. (1999) A Educação Espacial na América Latina e a Posição do Brasil no Contexto Regional. Cooperação internacional na área espacial. Parcerias Estratégicas, 7: 151-164.

Schappe R. \& Barbosa C. (2017) A Simple, Inexpensive Acoustic Levitation Apparatus. Physics Teacher, 55(1): 6-7.

Secco R.A. \& Sukara R.E. (2016) Indoor Microgravity Survey. Physics Teacher, 54(4): 213-215.

Sousa R. (2015) Construção de um experimento para o ensino de microgravidade com uso de vídeos. Dissertação (Programa de Pós-Graduação em Ensino de Física). Instituto Federal de Educação, Ciência e Tecnologia do Rio Grande do Norte, Natal, Rio Grande do Norte.

Temiz B.K. \& Yavuz A. (2014) Students' misconceptions about Newton's second law in outer space. European Journal of Physics, 35(4): 045004. http://dx.doi.org/10.1088/0143-0807/35/4/045004

Tenório T., de Melo L.R. \& Tenorio A. (2014) Séries televisivas de investigação criminal e o ensino de ciências: Uma proposta educacional. Revista Electrónica de Enseñanza de las Ciencias, 13(1): 73-96.

Vasconcelos F.E.O. \& Marranghello G.F. (2015) A Astronomia e o Ensino Médio politécnico (p. 107-163). In: Marranghello G.F. \& Lindemann R.H. (Orgs). Ensino de Ciências na Região da Campanha. Volume 1. São Leopoldo: Editora Oikos.

Vogt G.L. \& Wargo M.J. (1992) Microgravity: A Teacher's Guide with Activities. Secondary Level. Disponível em: https://files.eric.ed.gov/fulltext/ED360178.pdf (Acessado em: maio/2020). 
Vogt G.L. \& Wargo M.J. (1995) Microgravity: Teacher's Guide with Activities for Physical Science. Disponível em: https://files.eric.ed.gov/fulltext/ED406237.pdf (Acessado em: maio/2020).

Vreeland P. (2002) Investigating Microgravity. Science Teacher, 69(7): 36-40.

Wasiliew C.E., Souza J.A.V., Scolari L.M., Ramos L.A.M. \& Rizzatti M.R. (2002) Experiências clássicas com situação de microgravidade. Parte I, II e III. Salão de iniciação Científica. Livro de resumos. Porto Alegre: UFRGS.

Williams P.H. (1997) Teachers and Students Investigating Plants in Space. A Teacher's Guide with Activities for Life Sciences. Grades 6-12. Disponível em: https://files.eric.ed.gov/fulltext/E D406233.pdf (Acessado em: maio/2020).

Zhang Y., Richards S.E., Richards J.T. \& Levine H.G. (2016) The Use of Microgravity Simulators for Space Research ISS R\&D San Diego. Disponível em: https://ntrs.nasa.gov/archive/nasa/casi. ntrs.nasa.gov/20160013279.pdf (Acessado em: abril/2020). 